$\underline{\text { Erratum }}$

\title{
Erratum to: Minimally Invasive Surgery for Obscure Idiopathic Ileal Varices Diagnosed by Capsule Endoscopy and Double Balloon Endoscopy: Report of a Case
}

Hirotaka Konishi ${ }^{1}$, Shojiro Kikuchi ${ }^{1}$, Atsushi Miyashita ${ }^{1}$, Daisuke Ichikawa ${ }^{1}$, Hitoshi Fujiwara ${ }^{1}$,

Takeshi Kubota ${ }^{1}$, Toshiya Ochiai ${ }^{1}$, Yukinito Kokuba ${ }^{1}$, Satoru Yasukawa ${ }^{2}$, Akio Yanagisawa ${ }^{2}$, and Eigo Otsuji ${ }^{1}$

Departments of ${ }^{1}$ Surgery and ${ }^{2}$ Pathology, Kyoto Prefectural University of Medicine, 465 Kajii-cho, Kamigyo-ku, Kyoto 602-8566, Japan

Erratum to: Vol. 40: 1088-1092, 2010

The correct name of the ninth author should be given as SAtoru Yasukawa, not SAtoshi Yasukawa.

The online version of the original article can be found at http://dx.doi.org/10.1007/s00595-009-4180-9 